\title{
Analysis of Fluxion and Heat Transfer Performance of Honeycomb Thermal Shroud for Space
}

\author{
Ran Liu, Lei Zhang \\ Beijing Institute of Space Environment Engineering, China \\ *liuran_cast511@126.com
}

Keywords: thermal shroud; heat transfer enhancement; numerical simulation

\begin{abstract}
In order to improve the simulation efficiency of the space environment, a kind of honeycomb thermal shroud is designed. Using the fluid mechanics theory and the finite element method to do the numerical simulation for the fluxion and the heat transfer enhancement of the internal fluid in the honeycomb thermal shroud, this paper analyzes the effects of the structural parameters of the honeycomb thermal shroud on the fluxion and the heat transfer to determine the optimal honeycomb structural parameters. The results are shown as follows: the temperature uniformity can reach $\pm 2 \mathrm{~K}$ under the load conditions; the depth and spacing of the internal flow of the thermal shroud is an important factor affecting the fluxion and heat transfer in the thermal shroud; it is necessary to consider the heat transfer and resistance problem to determine the optimal thermal shroud structural parameters.; the optimal separation distance of the thermal shroud passage is $75 \mathrm{~mm}$ and the depth is $10 \mathrm{~mm}$.
\end{abstract}

\section{Introduction}

The spacecraft needs to conduct a full vacuum thermal test in the space environment simulation facility before launch to guarantee the reliability of the spacecraft design. The thermal shroud is the core component of the space environment simulation facility, which simulates the space cryogenic environment. Therefore, the heat transfer performance of the thermal shroud can directly influence the simulation effect of model test. At present, the space simulation facility often adopts the pipe-plate thermal shroud, and the heat transfer is strengthened in the way of welding fins in the round pipe ${ }^{[1-3]}$. Because the fin is the secondary outer surface of the circular steel pipe, when the heat transfer area is added for heat transfer enhancement, the thermal resistance of the heat transfer is also increased at the same time, which makes the thermal load capacity of thermal shroud's unit area of this structure low and the temperature uniformity of the thermal shroud is not easy to control.

In this study, a kind of honeycomb thermal shroud was designed with good low temperature performance of stainless steel as the thermal shroud material. This thermal shroud is installed with pin fins inside, which can enhance the fluid disturbance, reduce or destroy the continuous development of the thermal boundary layer, so as to strengthen the heat transfer. At the same time, the heat transfer medium has direct contact with the hot surface, all heat transfer surfaces are directly involved in the heat transfer surface, and the heat load capacity is relatively strong. Through the numerical simulation calculation of heat transfer performance of different structural plate thermal shrouds, the temperature distribution of the wall surface and the flow and heat transfer performance curves are obtained, so the optimal flow structure parameters can be determined.

\section{Geometrical models of thermal shroud}

The geometrical structure of the honeycomb thermal shroud is shown in Fig 1, and the two stainless steel plates have a certain height interlayer between them; In order to enhance the heat transfer, the cylindrical pin fins are fixed up in the middle of the two stainless heat transfer plates, and the pin fins are arranged evenly according to the certain separation distance. In the structural parameters of the thermal shroud, the depth of the flow passage $\mathrm{H}$ and the separation distance $\mathrm{L}$ have the biggest impact on the thermal shroud performance. To study the impact of the structural parameters of the thermal 
shroud on the thermal shroud performance, different geometrical parameters of depth and separation distance are selected, which are shown in the Tab I.
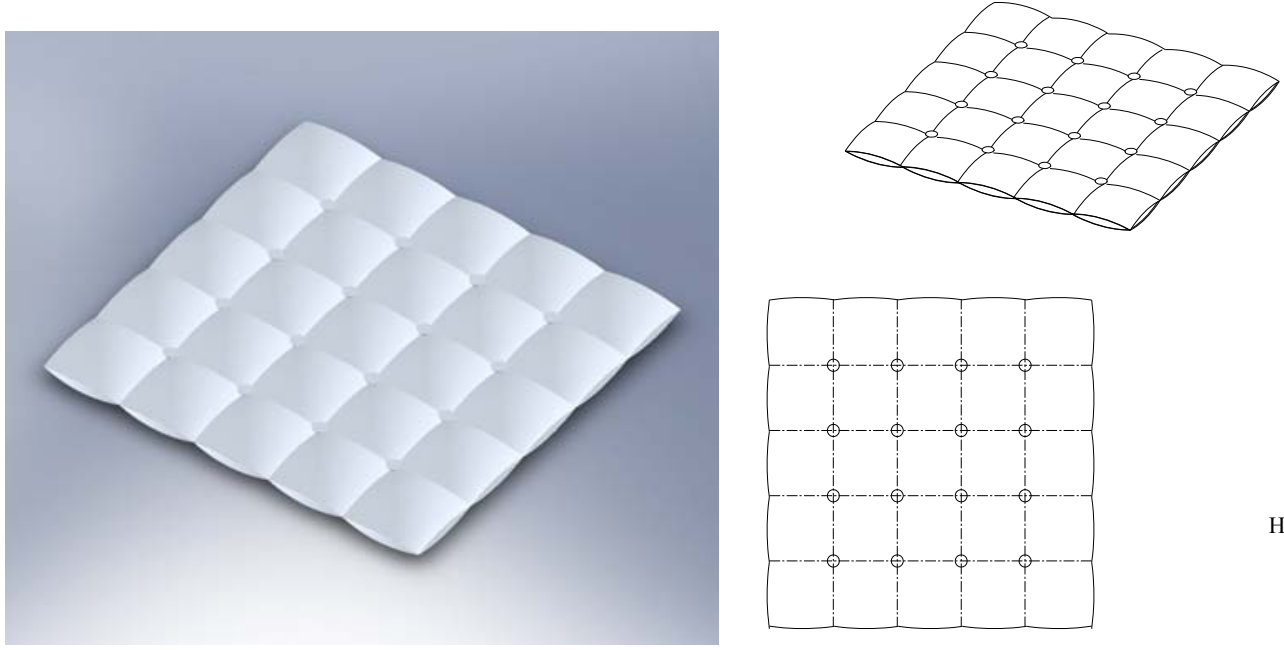

Fig1. Schematic diagram of structural unit of thermal shroud

Tabl Thermal shroud geometric parameter table

\begin{tabular}{|l|c|c|c|c|c|c|c|}
\hline Parameter & \multicolumn{4}{|c|}{ Separation distance fixed } & \multicolumn{4}{c|}{ Depth fixed } \\
\hline Depth $H(\mathrm{~mm})$ & 5 & 10 & 15 & 20 & \multicolumn{3}{c|}{10} \\
\hline $\begin{array}{l}\text { Separation distance } \\
L(\mathrm{~mm})\end{array}$ & \multicolumn{3}{|c|}{75} & 35 & 50 & 75 & 105 \\
\hline
\end{tabular}

\section{Simulation calculation and analysis}

\subsection{Calculation condition and simulation calculation}

Using the Gambit software to classify the geometric model and set the boundary condition type, and the Gambit generated file is imported into the Fluent software for simulation calculation. During the simulation, for the cooling medium, the thermal conductivity liquid is chosen. Taking the flow boundary conditions of the speed inlet and free outlet, the inlet velocity is set according to the study requirements and the temperature is $198 \mathrm{~K}$; the boundary condition of inner wall surface is set as the boundary condition of no-slip speed, $\mathrm{u}=0$ and the temperature distribution is subject to constant heat flow boundary condition. The equation $\mathrm{k}-\varepsilon$ of RNG model is used to calculate the turbulence model and the pressure-velocity coupling adopts the Simple algorithm. The discrete of each parameter adopts the Second Order Upwind and the criterion of the solution convergence is the relative residual $\mathrm{R} \leqslant 1$ $\times 10^{-5}$. After much iteration, the solution convergence is calculated and the simulation process is completed.

\subsection{Thermal shroud of different depths of flow passageway}

\subsubsection{Calculation results}

Temperature field of different flow passageway depths are shown in Fig 2.Nu and $\triangle \mathrm{P}$ curve is shown in Fig 3 and Fig 4. 

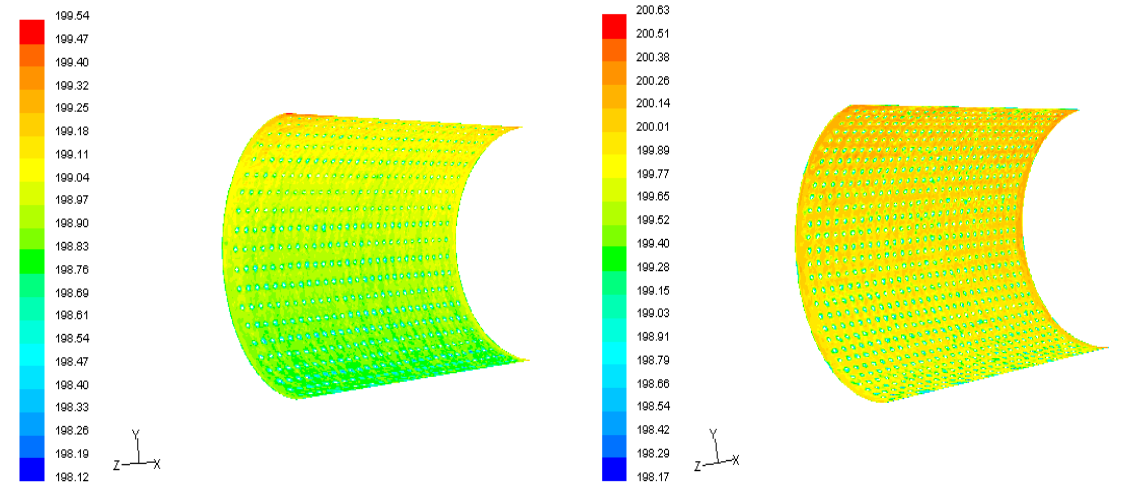

(a) $\mathrm{H}=5 \mathrm{~mm}$

(b) $\mathrm{H}=10 \mathrm{~mm}$
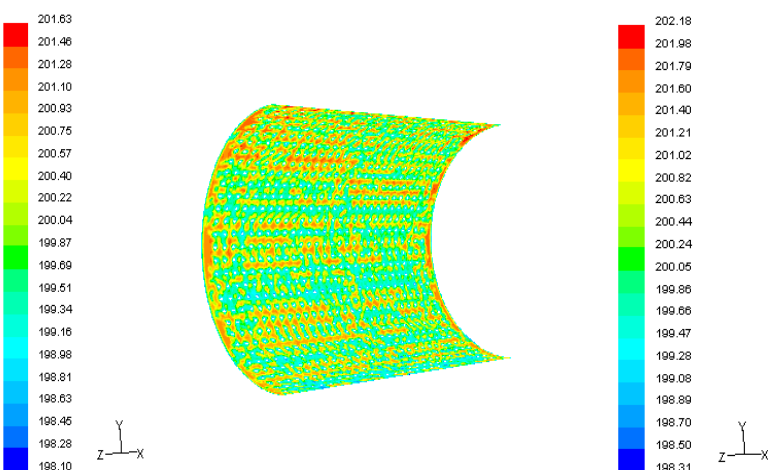

(c) $\mathrm{H}=15 \mathrm{~mm}$

(d) $\mathrm{H}=20 \mathrm{~mm}$

Fig2 Temperature field of different flow passageway depths

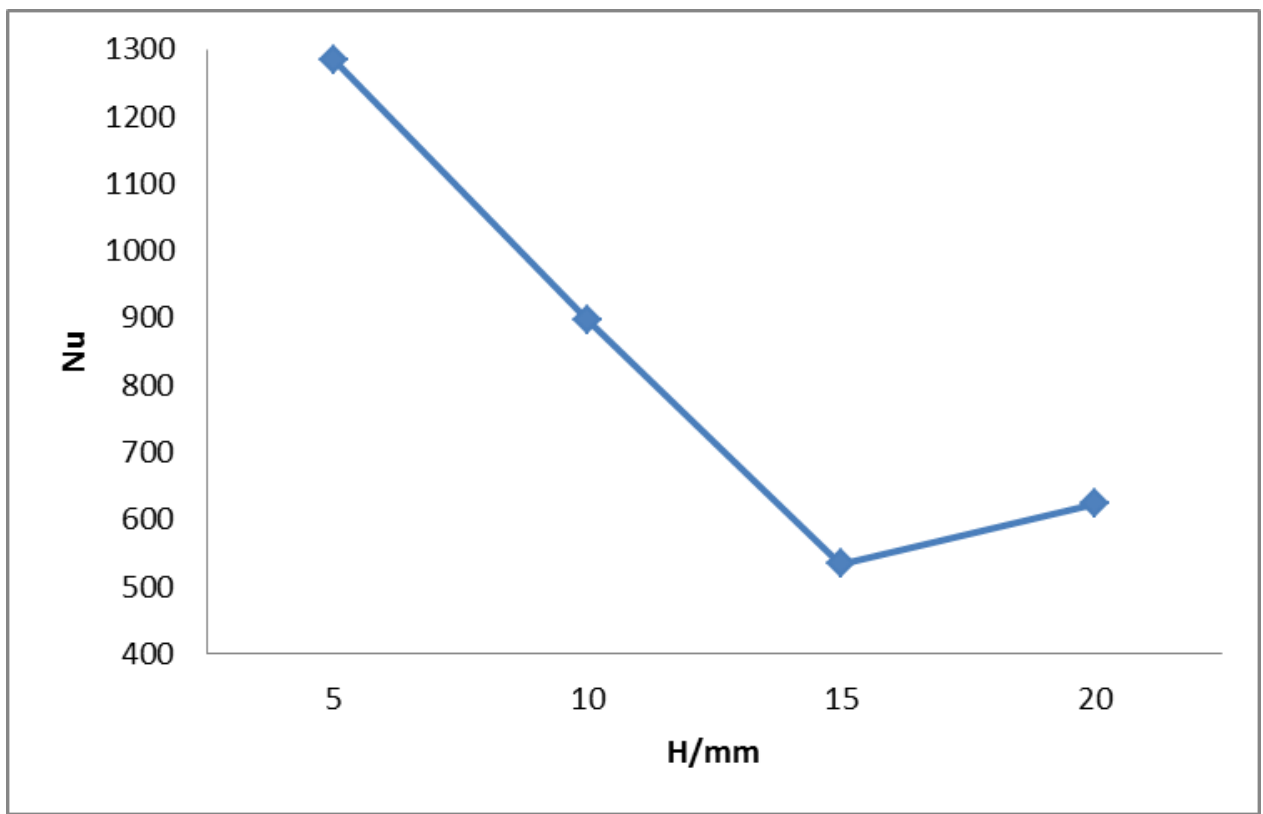

Fig 3 Heat transfer coefficient curve of different flow passageway depths 


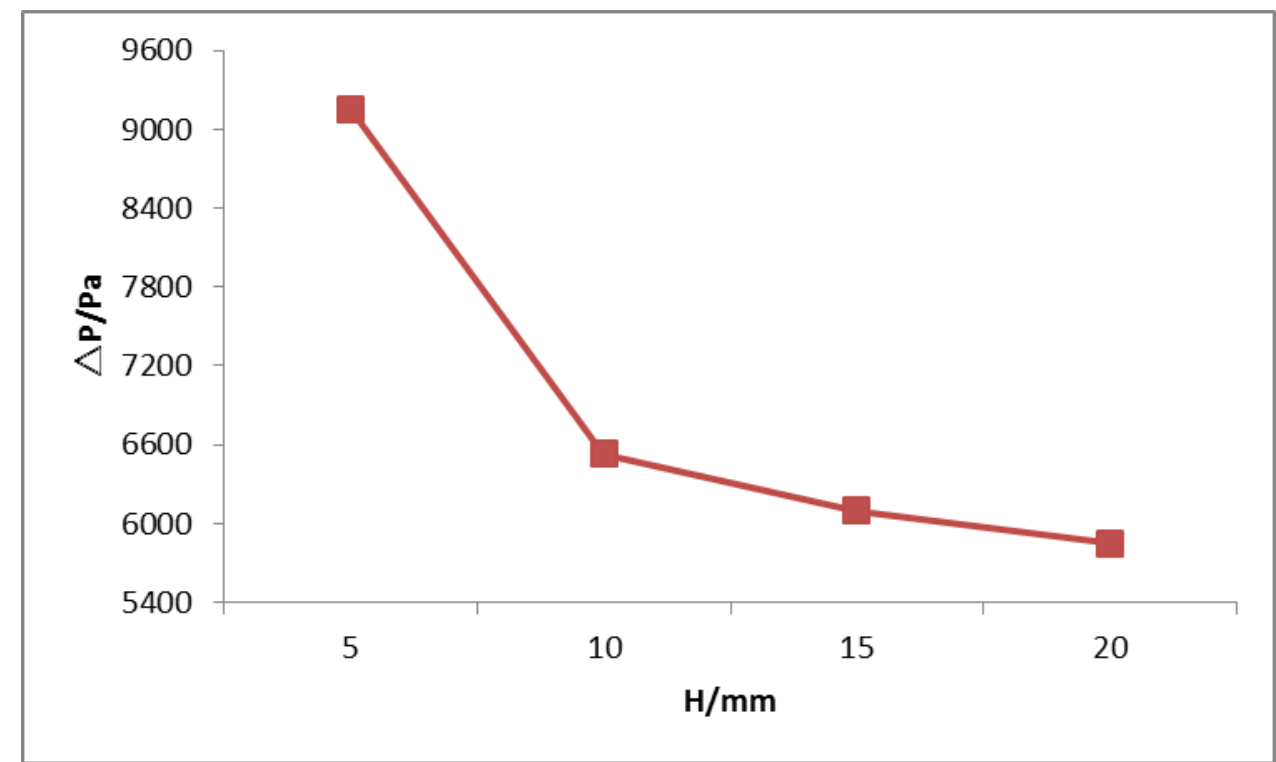

Fig 4 Pressure drop curve of different flow passageway depths

3.2.2 The impact of the flow passage depth on the heat transfer performance of the thermal shroud

It can be seen from Fig 2 that when the depth is $5 \mathrm{~mm}$ and $10 \mathrm{~mm}$, the uniformity of the thermal shroud temperature is $\pm 2 \mathrm{~K}$; when the depth is $15 \mathrm{~mm}$ and $20 \mathrm{~mm}$, the uniformity of the thermal shroud temperature is $\pm 3 \mathrm{~K}$. The influence of flow depth on temperature uniformity of thermal shroud wall is not obvious. This is because there are many small columns in the internal part of the thermal shroud, and the number of small cylinders in the thermal shroud with different depths is same, so the temperature uniformity has little difference.

From the Fig 3, it can be seen that with the increase of the flow passageway depth, Nu decreases and the heat transfer effects become bad. Under the condition of constant flow, the increase of flow depth can reduce the flow velocity of the internal fluid of the thermal shroud, resulting in the bad heat transfer effects. In the actual work of thermal shroud, the lower the flow depth, the more the tendency of scaling. If the flow depth $\mathrm{H}$ is too small, it may cause the fouling in the thermal shroud internal part. In addition, the smaller the flow depth, the larger the flow pressure loss $\triangle P$, which is shown in Fig 4 .

\subsection{Thermal shroud of different separation distances of flow passageway}

\subsubsection{Calculation results}

Temperature field of different flow passageway separation distances are shown in Fig 5.Nu and $\triangle$ $P$ curve is shown in Fig 6 and Fig 7. 

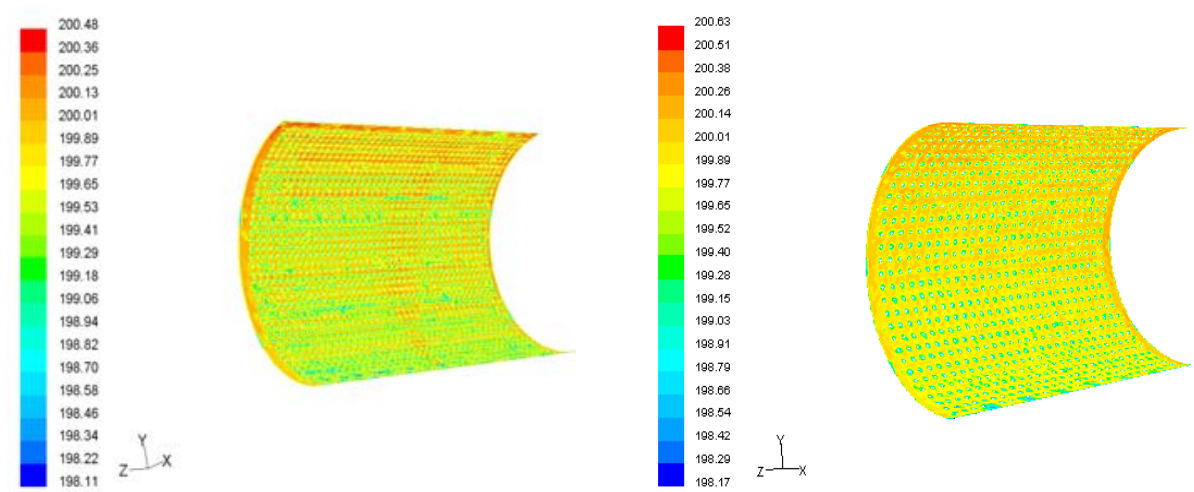

(a) $\mathrm{L}=35 \mathrm{~mm}$

(b) $\mathrm{L}=50 \mathrm{~mm}$
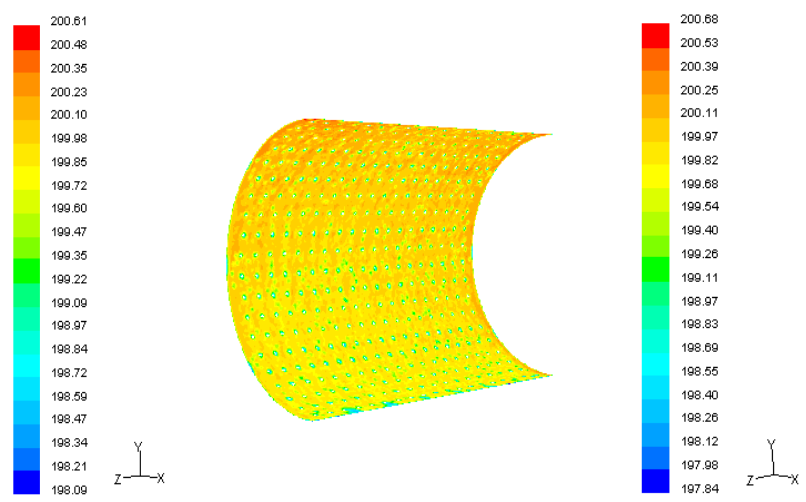

(c) $\mathrm{L}=75 \mathrm{~mm}$

(d) $\mathrm{L}=100 \mathrm{~mm}$

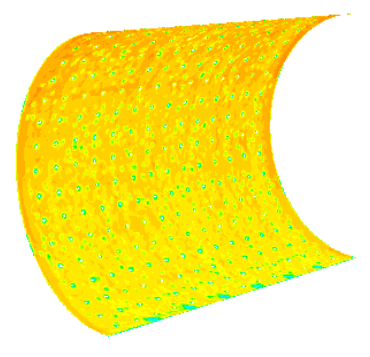

Fig 5 Temperature field of different flow passageway separation distances

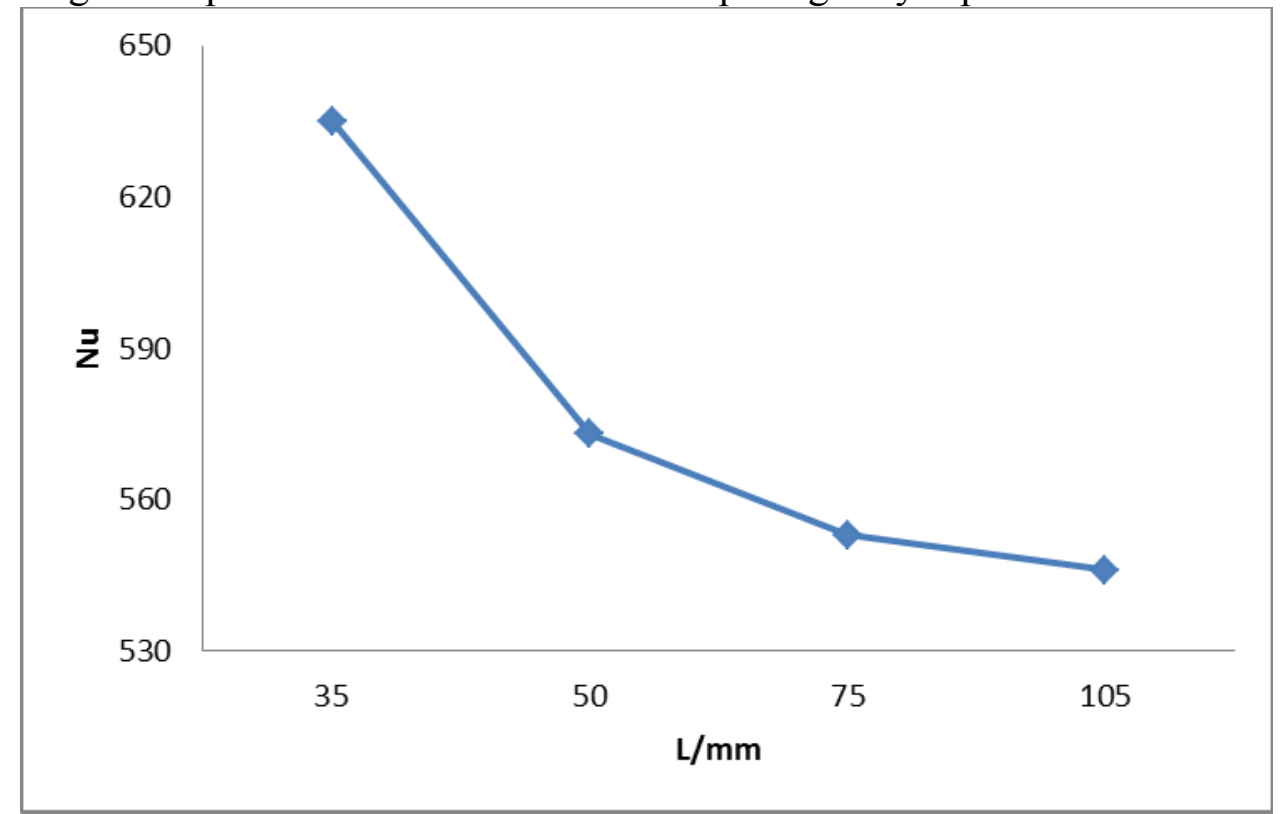

Fig 6 Heat transfer coefficient curve of different flow passageway separation distances 


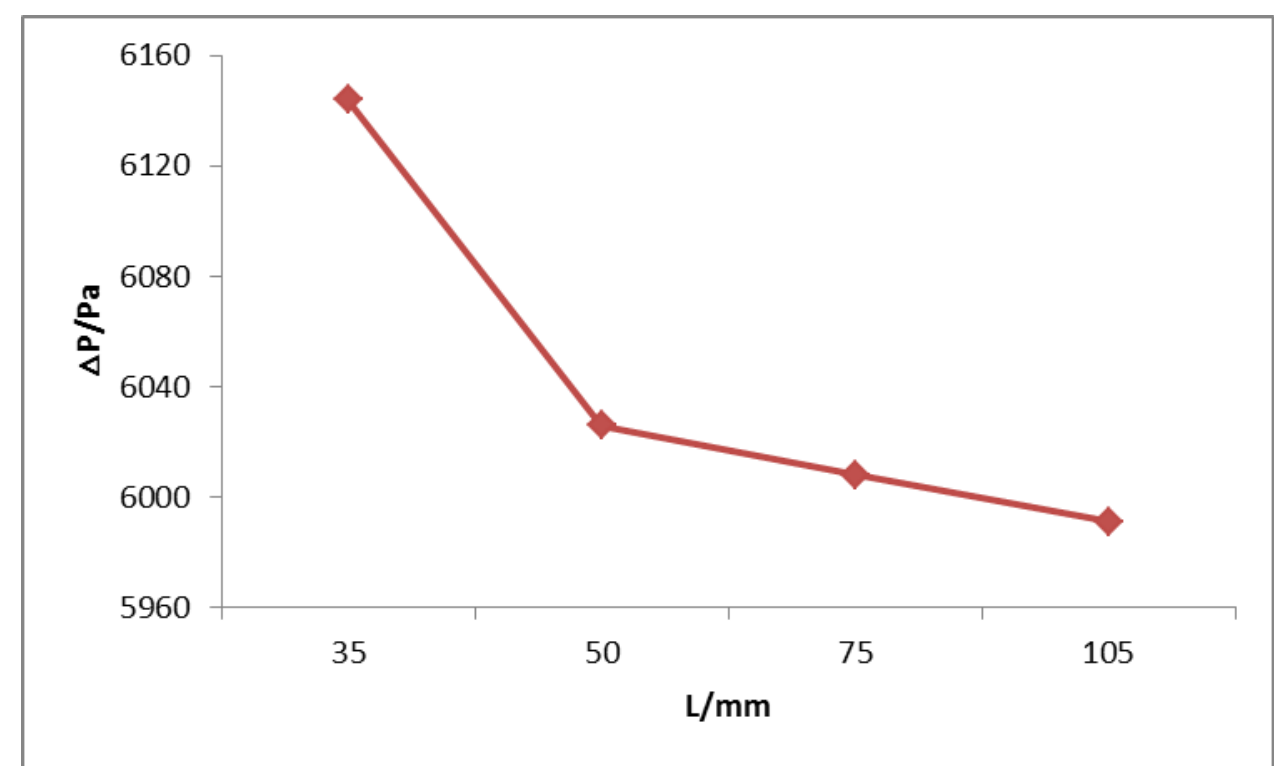

Fig 7 Pressure drop curve of different flow passageway separation distances

3.3.2 Impact of different separation distances of flow passageway on the heat transfer performance of the thermal shroud

From the Fig 5, it can be seen that the thermal shroud's temperature uniformity of different separation distances can reach $\pm 2 \mathrm{~K}$, and the influence of the channel spacing on the temperature uniformity of the thermal shroud wall is not significant. As the separation distance increases, the number of small columns in the thermal shroud decreases, and the turbulent flow decreases. However, due to the uniform distribution of the small columns under different separation distances of the flow passageway, the overall temperature distribution of the thermal shroud wall has not been affected.

As can be seen from Fig 6 and Fig 7, the distance between the flow channel is reduced from 100mm to $75 \mathrm{~mm}$. Both $\mathrm{Nu}$ and $\triangle \mathrm{P}$ are have increased, but the amplitude of the variation is not large; However, both $\mathrm{Nu}$ and $\triangle \mathrm{P}$ have increased significantly when the distance between the channel is reduced from $75 \mathrm{~mm}$ to $35 \mathrm{~mm}$. This is because as the flow channel spacing decreases, the number of the internal small cylinder of the thermal shroud increase and the turbulent flow function increases, which strengthens the heat transfer efficiency between the fluid and thermal shroud, and flow resistance also becomes bigger. When the number of the small cylinders reaches to a certain degree, the secondary reflux will appear and the heat transfer between the fluid and the wall is strengthened. However, the flow resistance is also increased. It can be seen from the above analysis that the heat transfer efficiency of thermal shroud cannot be improved by reducing the channel spacing independently, and the optimal channel spacing should be determined by combining heat transfer and resistance.

\section{Conclusion}

The honeycomb thermal shroud adopts the method of strengthening convective heat transfer effects to enhance the heat transfer effect, which is completely different from the traditional design concept of the tube-plate thermal shroud. The results of the simulation calculation show: the optimal temperature uniformity of the honeycomb thermal shroud under the load conditions can be $\pm 2 \mathrm{~K}$; the depth and spacing of thermal shrouds are important factors for heat transfer performance. Reasonable selection of flow structural parameters can improve heat transfer performance; it is necessary to combine heat transfer and resistance problem to determine the optimal flow structural parameters. The optimal separation distance of the thermal shroud's flow passageway is $75 \mathrm{~mm}$ and the depth is $10 \mathrm{~mm}$.

\section{Reference}

[1] Guee-Won Moon, Hyokjin Cho, et al.Shroud System Design for A Large Thermal Vacuum Chamber[C]// 49th Annual Technical Meeting and Exposition of the Institute of Environmental Science and Technology. 2003: 479-484 
[2] Watson R N, Proulx G F. A new thermal vacuum facility for Hughes space and communications at El Segundo California[C]//20th Space Simulation Conference the Changing Testing Paradigm. 1998: 113-130

[3] Govindan P, Satyanarayana M, Deviprasad Karnik, et al. Design and performance of $\varphi 0.6 \mathrm{~m}$ thermal vacuum chamber[C]//Proceedings of the 18th International Cryogenic Engineering Conference, 2000: 679-682 